\title{
PERCEPCIÓN DE APOYO Y MECANISMOS DE AFRONTAMIENTO EN MUJERES VÍCTIMAS DEL CONFLICTO ARMADO DEL MUNICIPIO SAN JUAN DE Nepomuceno, Bolívar, Colombia
}

\author{
Perception OF SUPPORT AND COPING \\ MECHANISMS IN WOMEN VICTIMS OF THE ARMED \\ CONFLICT MUNICIPALITY SAN JUAN DE \\ Nepomuceno, Bolívar, Colombia
}

Jorge Andres Martinez Merlo

Universidad Simón Bolívar. Colombia.

Martinez Merlo, J. (2020). PERCEPCIÓN DE APOYO Y MECANISMOS DE AFRONTAMIENTO EN MUJERES VÍCTIMAS DEL CONFLICTO ARMADO DEL MUNICIPIO SAN JUAN DE NEPOMUCEO, BOLIVAR, COLOMBIA. Revista Ene De Enfermería, 14(2). Consultado de http://eneenfermeria.org/ojs/index.php/ENE/article/view/1107 


\section{Resumen}

El objetivo de la investigación fue describir el apoyo percibido y los mecanismos de afrontamientos en las mujeres víctimas del conflicto armado que asisten a una fundación del Municipio de San Juan de Nepomuceno, Colombia. La metodología fue cualitativa de tipo fenomenológico, en el cual se usó como técnica de recolección la entrevista semi- estructurada individualizada. Los resultados evidencian que los mecanismos de afrontamiento utilizados por las mujeres víctimas del conflicto armado fueron el trabajo, la participación en talleres de ayuda psico-emocional y la religión. Además, se obtuvo una percepción de falta de apoyo familiar, social y gubernamental por parte de las entrevistados.

Palabras claves: Adaptación psicológica; Mujeres; Conflictos armados; percepción; apoyo social

\section{Abstract}

The objective of the investigation was to describe the perceived support and coping mechanisms in women victims of armed conflict attending a foundation of the Municipality of San Juan de Nepomuceno, Colombia. The methodology was qualitative of a phenomenological type, in which the individualized semistructured interview was used as the collection technique. The results show that the coping mechanisms used by women victims of armed conflict were work, participation in psycho-emotional assistance workshops and religion. In addition, a perception of lack of family, social and governmental support was obtained from the interviewees.

Keywords: Adaptation psychological; women; armed conflicts; Perception; Social Support 


\section{Resumo}

Percepção de apoio e mecanismos de enfrentamento em mulheres vítimas do conflito armado municipalidade San Juan de Nepomuceno, Bolívar - Colômbia

O objetivo da pesquisa foi descrever os mecanismos de apoio e enfrentamento percebidos em mulheres vítimas do conflito armado que freqüentam uma fundação sem fins lucrativos do Município de San Juan de Nepomuceno. A metodologia foi qualitativa do tipo fenomenológica, na qual a entrevista semiestruturada individualizada foi utilizada como técnica de coleta. Os resultados nostramo que alguns mecanismos de enfrentamento utilizados pelas mulheres vítimas do conflito armado foram trabalho, participação em oficinas de ajuda psicoemocional por meio de grupos de apoio e religião. Além disso, obteve-se uma percepção de falta de apoio familiar, social e governamental por parte dos entrevistados.

Palabras chaves: Adaptação Psicológica; mulheres; Conflitos Armados; Percepção; Apoio Socia 


\section{INTRODUCCIÓN}

Los conflictos armados desde hace tiempo han causado millones de víctimas, violando el derecho internacional humanitario, provocando muertes, genocidios, desplazamiento entre otros tipos de actos violentos (1).

Países como Sudán del Sur, Guatemala, el Salvador, Afganistán entre otros, han vivido los horrores del conflicto armado. En el caso de Sudán del Sur, a causa del conflicto armado se evidenciaron picos altos de violencia, siendo uno de los hechos más atroces, el genocidio de carácter étnico perpetrado por los milicianos árabes Janjaweed contra la población aborigen africana asentada en Darfur, al oeste de Sudán (2). De igual forma en Guatemala durante los años 60 se vivió una época oscura a causa de la oleada de ataques de la guerrilla y los contrainsurgentes del estado provocando una serie de secuelas en las personas por actos como las torturas, las desapariciones forzadas, las violaciones a los derechos humanos causando graves daños a la población, principalmente de las áreas rurales (3).

En el caso del salvador el conflicto armado entre el gobierno y los grupos insurgentes afectaron en gran parte a la población civil, siendo víctimas de violación de los derechos fundamentales a causa de hechos violentos como las ma- sacres y el desplazamiento forzado. De igual forma, en el caso de Afganistán hasta el momento también han dejado a su paso hechos violentos y degradantes como la tortura y el maltrato a la población (2).

En Colombia el conflicto armado interno ha sido uno de los hechos más crudos de la historia del país, siendo este uno de los causales de muchas muertes de mujeres, hombres y niños. A su vez, este ha sido uno de los conflictos internos más largos en el mundo por casi más de 60 años (4-6).

En el 2014 las cifras señalaban que en Colombia el número de víctimas registradas era superior a siete millones, es decir el $15 \%$ de la población de Colombia ha sufrido de violencia (7). Respecto a los Montes de María, subregión donde pertenece el municipio de San Juan de Nepomuceno, unos de los actos más recordados es la masacre perpetrada en el corregimiento del Salado en el 2000 , donde ejecutaron extrajudicialmente a 46 campesinos dentro de los que se encontraban nueve mujeres, una de ellas menor de edad $(8,9)$.

Diversos autores señalan que el conflicto armado interno Colombiano ha causado en la población civil, afectaciones físicas, sociales y afectivas en los individuos afectados a corto y a largo plazo ( 10-12); de este modo en el estu- 
dio realizado por Médicos sin Fronteras (13), reveló que la violencia es, entre otros factores estudiados, el evento que más afecta la salud mental de la población civil, con diagnósticos habituales como la depresión (25\%), ansiedad (13\%), trastorno mental (11\%) y estrés postraumático (8\%). Por otro lado, otro estudio evidencio que el $90 \%$ de los entrevistados presentaron síntomas de depresión y en el $60 \%$, valores conclusivos de ansiedad, depresión y propensión a desarrollar síntomas de estrés postraumático (14).

No obstante; las fuentes de apoyo tanto familiar, social como gubernamental son fundamentales para la reducción de secuelas y el manejo de las afecciones psicosociales causadas por la violencia. En un estudio realizado por (15) se encontró que el apoyo percibido de la familia ayuda a disminuir la frecuencia e intensidad de la violencia, en tanto que el apoyo percibido de los amigos tiene un efecto en la reducción del malestar en las participantes. Así mismo, un estudio realizado por (16) encontraron que, en la muestra recogida en el municipio de Trujillo, se pudo encontrar una asociación significativa entre el apoyo social percibido y el bienestar psicológico. Ahora bien, respecto al apoyo desde el contexto gubernamental, diferentes han sido los mecanismos implementados para la reparación a la víctima y la estabilidad psicosocial tanto del individuo como su familia; entre esos la ley 1448 (17) "Por la cual se dictan medidas de atención, asistencia y reparación integral a las víctimas del conflicto armado interno y se dictan otras disposiciones" ; a través de esta, se diseñó un programa de atención psicosocial, el cual busca mitigar, superar y prevenir los daños e impactos a la integridad psicológica y moral, al proyecto de vida y la vida en relación, generados a las víctimas, sus familias y comunidades por las graves violaciones de Derechos Humanos e infracciones al Derecho Internacional Humanitario .

Desde el contexto individual para enfrentar los hechos traumáticos, violentos o impactantes, existen diversos mecanismos de afrontamiento, los cuales son un conjunto de recursos psicológicos que el sujeto pone en marcha para hacer frente a situaciones estresantes (18). En Colombia estudios señalan que algunas de las estrategias de afrontamiento utilizadas son el uso del afrontamiento religioso, reevaluación positiva, estrategias de distracción y uso de cualidades personales (19).

Por tal razón, el objetivo de este estudio es describir la percepción de apoyo y los mecanismos de afrontamientos en las mujeres víctimas del conflicto 
armado que asisten a una fundación sin ánimo de lucro del Municipio de San Juan de Nepomuceno.

\section{MATERIAL Y MÉTODO}

\section{PARTICIPANTES}

La población objeto de estudio estuvo conformada por 10 mujeres víctimas del conflicto armado que asisten a una fundación sin ánimo de lucro en el municipio de San Juan de Nepomuceno en el departamento del Bolívar- Colombia. la selección de la población se realizó mediante muestreo no probabilístico a conveniencia; además, debían cumplir con los siguientes criterios: Haber sido víctimas directas o indirectas de algún hecho violento durante el conflicto armado, mujeres entre edades de 18 a 55 años o más y haber recibido y aceptado la invitación a participar de la investigación con previo consentimiento informado firmado. Así mismo, dentro de los criterios de exclusión aquellas mujeres que, por alguna circunstancia, hecho o motivo no se encontrara en su vivienda durante la recolección de la información, también aquellas mujeres que hayan sido diagnosticadas con alguna enfermedad mental.
Tal como se indicó, la recolección de los datos se realizó a través de una entrevista semi-estructurada la cual contaba con 4 preguntas orientadoras enfocadas en dar respuesta al objeto de investigación; cada entrevista tuvo una duración promedio de 30 minutos. Esto permitió explorar las respuestas sociopolíticas de las mujeres objeto de estudio, respecto a los mecanismos de afrontamiento.

RECOLECCIÓN Y ANÁLISIS DE

\section{LOS DATOS}

Se contactó a las mujeres que habían manifestado interés en participar en las entrevistas que se realizarían en la fundación sin ánimo de lucro, a las cuales se les realizó, previo consentimiento informado, una entrevista semiestructurada individualizada. Las entrevistas fueron grabadas en un dispositivo de audio en formato mp3 player. Posteriormente, se realizó la transcripción de dichos audios, luego se procesó la información a partir de dos procesos: comprensión y síntesis de la información suministrada por los participantes. Para el análisis de la información se clasificaron en tablas los descriptores, junto con la transcripción textual de la información asignándoles colores y estableciendo un código por descriptor. Se tuvo en cuenta la similitud conceptual en cada uno de ellos ayudado con las notas de campo. 
De igual forma, se mantuvo secuencialidad en la referencia numérica, para efectos de organización de la información y el análisis contextual y de patrones; de esta manera, se les asignó a las participantes la letra E (entrevista), seguida del número 1; así mismo para cuestiones de confidencialidad y protección de la identidad de la población objeto de estudio, se asignó la letra $P$ (participante) seguida de un número del 1 al 10 que identifican a cada individuo. Ejemplo: "En estos momentos me siento bien, gracias a Dios" .... E1P1: Entrevista 1, Participante 1.

\section{CONSIDERACIONES ÉTICAS}

Para la realización de este estudio, se tuvo en cuenta las consideraciones éticas en la investigación como fue la resolución nº 8430 de 1993 (23). y los principios éticos para la investigación médicas en seres humano postuladas en la declaración de Helsinki (24). Además, este estudio fue aprobado por el comité de ética de la Universidad Simón Bolívar bajo el No PRO-CEI-USB-CE-0243-00, mediante acta de aprobación de proyectos $\mathrm{N}^{\circ} 00194$ del 14 de mayo del 2018.

\section{RESULTADOS}

Respecto a los datos sociodemográficos, las edades de las participantes oscilan entre 23 a 60 años. Así mismo, según el grado de escolaridad se identificó que en mayor proporción tenían primaria incompleta. En cuanto a la actividad laboral, la mayoría se dedicaba a oficios del hogar (Ama de casa) y otras se dedicaban a actividades informales (Oficios varios). Por otro lado, todas manifestaron tener hijos (2 a 3 hijos).

De acuerdo con los pasos de interpretación y análisis aparecieron unas sub-categorías: Percepción de apoyo después del hecho violento y mecanismos de afrontamiento, pertenecientes a la categoría: Experiencias vividas durante el conflicto armado.

PERCEPCIÓN DE APOYO DESPUÉS DEL HECHO VIOLENTO

En esta categoría se examinó si luego del hecho violento ocurrido, las mujeres objeto de estudio de alguna manera u otra se habían sentido apoyadas sea por su familia, vecinos, comunidad o por el gobierno. De acuerdo al apoyo percibido después del hecho violento, las mujeres manifestaron no sentirse apoyadas por sus vecinos, y algunos cambios respecto a las tradiciones o actividades que realizaban en la comunidad.

Antes por ejemplo no tenía bastimento, iba donde el vecino, préstamelo que ahorita que venga mi papa o algo te lo devuelvo y ellos te prestaban... ahora todo es comprado, ahora no te ayudan. (EIP1) 
Los vecinos pa decir pa 31, semana santa se reunían en el rancho a hacer el mote, hacer dulce y para 31 a matar (res, pollo, cerdo) pa hacer pa los pasteles, y ya después del hecho ocurrido no, entonces afecta esas cosas afectan. (E1P5)

Por otra parte, se pudo evidenciar que algunas mujeres recibieron en un determinado tiempo apoyo por parte del gobierno, tanto económico como psicológico. Sin embargo, la percepción de las mujeres fue falta de apoyo gubernamental después de los hechos violentos ocurridos.

Ayuda así que uno diga ayuda no, porque el gobierno solo me da cada 5 meses o cada 6 meses me dan una ayudita, que la llaman humanitaria. (E1P1)

Fui a declarar en el 2009, y a mí nunca me quisieron atender, en el 2011 volví a ir y tampoco, del 2014 pa acá protección social me ayuda con 180mil y me mandan mercado cada 5 o 6 meses eso es todo lo que da. Les dije que necesitaba psicólogo, pero nunca... me dijeron que mandarían, pero jumm nada. (E1P2)

Pues... yo fui a las oficinas y me dijeron que ya no ni nada, de ayuda económica ni nada. (E1P3)

Apoyo Así en abundancia, no, mi esposo le dieron una plata, le vinieron dos veces ayuda, lo indemnizaron y eso, por un lado, nos apoyado en eso. (E1P6)

El gobierno no nos ayudó, el gobierno primero nos mandó a la cruz roja, a los 8 días estaba en San Juan, y nos dio una ayuda de alimentación, nos dieron para dormir unas colchoneticas, sabanas, jabones y así elementos básicos, eso fue el apoyo que nos dieron. (E1P8)

Siento que otra vez estoy volviendo a lo mismo, con el temor y miedo. Actualmente no estoy recibiendo nada de apoyo psicológico. (E1P9)

Finalmente, referente al apoyo familiar algunas señalaron que luego del hecho, sus familiares no la ayudaron y sus parejas la abandonaron, quedando totalmente vulnerable en el aspecto económico.

Ni la familia ni el papa de los pelaos me apoyan, porque él me abandono, cuando yo me fui de allá, he tenido que sacar adelante a mis 7 hijos. (E1P1)

Una de las mujeres manifestó haber sido víctima de violación sexual, situación que describe como una emoción fuerte. A raíz de dicha situación, decide denunciar el hecho. Sin embargo, manifiesta no haber sido apoyada por su esposo y ocurre todo lo contrario, la agrede psicológicamente.

La emoción más fuerte que yo sentí de todo lo que me hicieron fue la violación. El papa de mis hijos, yo le dije 
que quería denunciar eso, entonces me dijo como que te gusto (la violación) porque quieres denunciar pa que vuelvan y te hagan peor, entonces, el como que piensa que me gusto y quiero que vuelvan. (E1P2)

Por otro lado, una de las mujeres manifestó tener problemas con su esposo en el aspecto sexual debido a la violación a la cual fue víctima durante el hecho violento. Situación a la cual no se sintió comprendida ni apoyada por su pareja.

Yo durante eso que paso fui violada, actualmente pues tengo una pareja, y no ha tenido comprensión, y eso me afecta. Sabiendo el mi caso, muchas situaciones que me pasaron, siento que a veces no tengo el apoyo que quiero por parte de él. Al momento de intimidad con mi esposo, hay momentos que me vuelven del pasado esos recuerdos y me deprimo. (E1P3).

Una de las entrevistadas manifiesta que fue abandonada por su esposo, porque cuando ocurrieron los hechos él quería seguir viviendo en el mismo lugar y ella debido al temor y miedo decidió salir del campo e irse a vivir al pueblo.

Cuando paso eso del desplazamiento vivía con un señor, pero ya no vivo con él porque cuando paso eso, él quería que yo me fuera pa allá donde paso eso, y yo decía que no me iba para allá. (E1P7)

Mecanismos de afrontamiento

De acuerdo a esta categoría, se evidencio que el trabajo para las mujeres víctimas del conflicto armado fue un mecanismo de afrontamiento que les ayudo a manejar las consecuencias de la violencia.

Pues, mecanismos o cosas que hice para afrontar eso, trabajar, nada más trabajar. (E1P1)

Pues jum para afrontar eso toca es trabajar y trabajar, ahora mismo mi esposo ya no trabaja, porque le quedo una discapacidad se puede decir física, porque por un problema en la mano. (E1P4)

Trabajar y aprender lo que no sabía, para sobrevivir, hacer pasteles, boIlos. (E1P5)

Pues, nada hay en la casa, se guimos adelante trabajando.... Para afrontar eso. (E1P6)

Bueno, buscar trabajo aquí en San Juan eso era lo que tenía que buscar, en los primeros días aja... como para manejar la situación dura pa comer y eso. (E1P8)

Así mismo, también señalaron que los talleres de ayuda psico-emocional a través de grupos de apoyo también les ayudo significativamente a afrontar las situaciones en el aspecto psicológico 
que aún estaban presente después del hecho violento. El conocer la situación de otras mujeres con situaciones aún más graves y que siguieron adelante, las hacía sentirse con deseos y motivaciones de auto-superarse.

Aquí (Fundación), trajeron psicólogos, nos ponían hacer dinámicas y así, para olvidar, nos llevaron para Bogotá, yo vi que, ósea... eso nos ayudó mucho, había victimas que habían perdido hasta 15, 20 familiares y así hermanos hijos sobrinos, y yo decía bueno si yo perdí nada más 2 personas, como que... yo tengo que luchar por mis hijos. (E1P1)

cuando vine aquí a la fundación he sentido un apoyo, y estando aquí en la fundación, la amiga que te dije que tenía, la que viste allá de ojito enfermito, ella me veía llorando me encontraba llorando, y me decía vámonos pa alguna parte, y veníamos aquí (fundación), nosotras nos poníamos hablar, eso yo sentía mucho apoyo aquí. (E1P2)

Bueno aquí en la fundación tengo mis compañeras, con las que tengo confianza, trato desahogarme, contarles mis problemas y así. Gracias a la organización hemos recibido ayuda psicosocial, la verdad que sí. (E1P3)

Me fui conociendo con las personas aquí (Fundación), había una facilitadora que nos hacían talleres y así, ella ya no viene aquí, se retiró, nos orientaban y eso. (E1P7)

Hemos tenido psicólogos, PASF$\mathrm{VI}$, nos dieron psicólogos, yo tengo 2 años apenas que salí del psicólogo. (E1P8)

Aquí de la fundación me han llevado a Bogotá a recibir capacitaciones, y esto me ha ayudado a afrontar un poco eso que siento y así. (E1P10)

No obstante, algunas mujeres señalaron la religión como mecanismo de afrontamiento y superación de la situación ocurrida. El aferrarse a Dios y tener fe las ayudo en su día a día, y las fortaleció para seguir adelante, ellas y sus hijos.

Primero pedirle mucho a Dios, cuando estoy en momentos así difíciles, de crisis. (E1P3)

Yo me aferre a Dios, me metí a un grupo de la iglesia católica me consagre, empecé a pedirle a Dios que me ayudara con ese rencor que sentía con esa gente, eso es malo odiar. (E1P9)

\section{DISCUSION Y CONCLUSION}

Los resultados obtenidos en este estudio respecto a la percepción de apo- 
yo social de las mujeres víctimas del conflicto armado que asisten a la fundación sin ánimo de lucro en el municipio de San Juan de Nepomuceno, Bolívar; señalaron una ausencia de ese apoyo y algunas tradiciones o actividades sociales que antes del hecho violento realizaban, habían desaparecido. Estos resultados al compararlos con los obtenidos en el estudio realizado por (16) mostraron discordancia, puesto que, en su caso, las victimas manifestaron haber percibido un apoyo efectivo de sus redes sociales en el afrontamiento de las situaciones derivadas del conflicto armado. Algunos autores señalan que las personas que atraviesan por situaciones no deseables a menudo pueden mantener un nivel de ajuste psicológico adecuado sí disponen además de apoyo social (25).

Respecto a la percepción de apoyo familiar, las victimas señalaron falta de apoyo, debido que al poco después del hecho violento sus parejas las abandonaron, en el caso de las víctimas de violación sexual tenían problemas al tener intimidad o relaciones sexuales con sus parejas, situación con la cual se sentían poco entendidas o comprendidas por su cónyuge. Además de esto, se evidencio por parte de la pareja expresiones culpabilizadoras hacia la víctima. Estos resultados coinciden con el estudio de Boira, Carbajosa y Méndez (26) en donde obtuvieron que, respecto a la familia, su papel en relación con el apoyo que brinda a la víctima es en ocasiones ambivalente e incluso puede llegar a ser culpabilizador.

Por otro lado, se evidencio que los mecanismos de afrontamientos utilizados por las participantes fueron el trabajo, asistir a grupos de apoyo y la religión. Esta serie de mecanismos las ayudo a manejar las consecuencias de la violencia, mencionaron que también les ayudo a afrontar las situaciones en el aspecto psicológico que aún estaban presente después del hecho violento; el conocer los casos de otras mujeres con situaciones aún más graves y que siguieron adelante, las hacía sentirse con deseos y motivaciones de auto-superarse. El aferrarse a Dios y tener fe las ayudo en su día a día, y las fortaleció para seguir adelante, a ellas y sus hijos. Estos resultados coinciden respecto a la religión como mecanismo con el estudio realizado por Hewitt et al (27) donde evidenciaron que la población de víctimas participante utiliza en mayor medida las estrategias de afrontamiento de esperar que las cosas se arreglen solas y la religión. También guardan concordancia con los estudios de Di-Colloredo, Aparicio, \& Moreno; Londoño et al y Vasconcelles (28-30) en donde evidenciaron que las 
personas expuestas a situaciones violentas, buscan apoyo en el uso de la religión.

En cuanto a los resultados obtenidos referente al trabajo como mecanismo de afrontamiento, guardaron coincidencias al compararlo con el estudio realizado por (19) donde identificaron que para las victimas era fundamental como estrategia de afrontamiento enfocada en el problema era conseguir un empleo, siendo la mayoría vendedores informales. Así mismo, conocer los casos de otras mujeres con situaciones aún más graves y compararse, es una estrategia de afrontamiento que de cierto modo las hacía sentirse mejor (19).

Como conclusión, es importante enfatizar los esfuerzos desde una perspectiva transdisciplinar, a fortalecer los vínculos sociales y familiares en las víctimas del conflicto armado. Puesto que, son el primer eslabón que busca la víctima para re-fortalecerse y buscar apoyo a su situación psico-emocional. En este aspecto, el estado tiene un papel transcendental. Aunque son diversos las leyes, modelos y demás mecanismos legales que ha interpuesto para la reparación a la víctima del conflicto armado; se hace necesario evaluar el impacto de la atención psicosocial integral, en busca de respuestas a la percepción negativa respecto al apoyo gubernamental tanto económica como emocional por parte de las víctimas. En cuanto a los mecanismos de afrontamiento es importante que las intervenciones se realicen colectivamente en la población afectada, con el fin de fortalecer los recursos de afrontamiento desde una perspectiva relacional; además, se deben implementar estrategias educativas orientadas a promover conductas agenciadoras de autocuidado en el aspecto psicosocial, con lo cual la victima pase de ser un agente pasivo en espera de ayuda, a un agente activo para el manejo de las afecciones psicosociales teniendo en cuenta los medios que dispone. 


\section{BiBLIOGRAFÍA}

1. Acevedo Suárez, A y Rojas Castillo, Z. (2016). Generalidades del conflicto, los procesos de paz y el posconflicto. Revista de la Facultad de Derecho y Ciencias Políticas, 46(124), 33-45. Retrieved February 04, 2019, from http://www.scielo.org.co/ $\mathrm{s}$ c i e l o . p h p ? script=sci_arttext\&pid=S0120-3886201600010000 $3 \& \operatorname{lng}=e n \&$ tlng=es.

2. Jaramillo, J. (2015). Perspectivas comparadas de la presencia de la policía durante el conflicto y el postconflicto: los casos de Guatemala, el salvador, Irlanda del norte, Afganistán y república del sur de Sudán. Revista de Relaciones Internacionales, Estrategia y Seguridad, 10(1):181-202. https:// doi.org/10.18359/ries.368

3. Monroy, M. (2003). Psicotrauma en sobrevivientes de guerra en Guatemala. Med. leg. Costa Rica, 20(2): 87-96. Available from: http://www.scielo.$\mathrm{s}$ a $. \mathrm{c} / \mathrm{s} \mathrm{c}$ i $\mathrm{e} / \mathrm{o}$. $\mathrm{p} \mathrm{h} \mathrm{p}$ ? script=sci_arttext\&pid=S1409-0015200300020000 $9 \& \operatorname{lng}=e n$

4. Calderón, J. (2016). Etapas del conflicto armado en Colombia: hacia el posconflicto. Revista de estudios Latinoamericanos, 62(1): 227-257. Recuperado en 06 de noviembre de 2018, de http:// w w w. s cielo.org. m x/scielo.ph p ? script=sci_arttext\&pid=S1665-8574201600010022 $7 \& \operatorname{lng}=e s \& t \operatorname{lng}=e s$

5. Obando, LM., Vizcaya, YC y Fernández, MC. (2016). Conflicto armado, problemática de poderes que ha vulnerado a la sociedad colombiana. Revista Katharsis, 21 (1): 385-417. Recuperado en 15 de enero de 2019, de http://revistas.iue.edu.co/ revistasiue/index.php/katharsis/article/view/ 774/1065 https://doi.org/10.25057/25005731.774

6. Ayala, T y Osorio, G. (2016). La mujer como víctima y actor del conflicto armado en Colombia. Perspectivas, 1(1): 73-80

7. Moreno, M y Díaz, M. (2015) Posturas en la atención psicosocial a víctimas del conflicto armado en Colombia. Rev. Ago. USB, 15(2): 325-585

8. Quintero Márquez, V., Otero Bahamón, S y Bolívar Ramírez, I. El abordaje del homicidio sexual en contexto de conflicto armado: El caso de la masacre del Salado. 2011. Análisis Político, 24(71), 109128. Retrieved November 06, 2018, from http:// www.scielo.org.co/scielo.php? script=sci_arttext\&pid=S0121-4705201100010000 $6 \& \operatorname{lng}=e n \&$ tlng=es.

9. Prada-Sanmiguel, A. (2016). Comprensión de la responsabilidad política de los actores armados en el conflicto interno colombiano: la masacre de El Salado 2000. Rev.latinoam.cienc.soc.niñez juv, 14(2): 1537-1548. Available from: http://www.scielo.org.co/scielo.php?script=sci_arttext\&pid=S1692-
715X2016000200045\&lng=en. http://dx.doi.org/ 10.11600/1692715x.14244010915.

10.Centro nacional de memoria histórica. (2013). ¡Basta ya! Colombia: memorias de guerra y dignidad. Bogotá: Imprenta Nacional. Recuperado de http://www.centrodememoriahistorica.gov.co/descargas/informes2013/bastaYa/basta-ya-memoriasguerra-dignidad-new-9-agosto.pdf

11.Lira, E (2010). Trauma, duelo, reparación y memoria: atención psicosocial del sufrimiento en el conflicto armado. Lecciones aprendidas. Revista universidad de los Andres, 36, 14-28.

12.McDonald, L. (2010). Psychosocial rehabilitation of civilians in conflict- affected settings. En E. Mertz (Ed.), Trauma rehabilitation after war and conflict, community and individual perspectives (pp 215-245). USA: Springer.

13. Médicos sin fronteras Colombia (MSF). (2017). A la sombra del proceso: Impacto de las otras violencias en la salud de la población colombiana. [cited 2019 Feb 04]; Disponible en: https:// arhp.msf.es/sites/default/files/MSF_Report-Colombia-Aug2017_SP.pdf

14.Revista semana. (2015). Conflicto y salud mental las heridas invisibles de la guerra. [Citado 22 jun. 2017]. Disponible en: http://especiales.semana.com/especiales/conflicto-salud-mental/

15.Rodríguez Hernández, R y Ortiz Aguilar, L. (2014). las fuentes de apoyo social y su impacto en la violencia y malestar en mujeres maltratadas. revista internacional de ciencias sociales y humanidades, sociotam, xxiv (2), 199-218.

16.Vásquez, J., Echeverri, M., Moreno, J., Carrasco, N., Ferrel, F Y Ferrel, L. (2018). El apoyo social percibido por las víctimas del conflicto armado en Colombia. Revista el ágora USB, 18(2), 362-373. Recuperado en 10 de enero de 2019, de https:// revistas.usb.edu.co/index.php/Agora/article/view/ 3387

17. Ministerio de salud y protección social colombiano. Atención psicosocial a víctimas. [citado 09 May. 2018].Disponible en: https://www.minsalud.gov.co/proteccionsocial/Paginas/Victimas_Atenc_Psicosocial.aspx

18.Macías, M., Madariaga Orozco, C., Valle Amarís, M., y Zambrano, J. (2013). Estrategias de afrontamiento individual y familiar frente a situaciones de estrés psicológico. Psicología desde el Caribe, 30 (1), 123-145.

19.Zuluaga, L. C. (2016). Estrategias de afrontamiento en un grupo de desplazados internos en la ciudad de Bogotá. Revista de Psicología Universidad de Antioquia, 8(2), 71-86.

20.Martínez, F. (2012). Fenomenología como método de investigación: Una opción para el profesional de enfermería. Enf Neurol (Mex) 11 (2): 98-101. http:// www.medigraphic.com/pdfs/enfneu/ene-2012/ ene122h.pdf 
21. Robles, B. (2011). La entrevista en profundidad: una técnica útil dentro del campo antropofísico. Cuicuilco, 18(52), 39-49. Recuperado en 24 de enero de 2019, de http://www.scieI o. o r g. m x / s c i e l o.p h p ? script=sci_arttext\&pid=S0185-1659201100030000 $4 \& \operatorname{lng}=e s \& t \operatorname{lng}=e s$.

22.Díaz-Bravo, L., Torruco-García, U., Martínez-Hernández, M y Varela-Ruiz, M. (2013). La entrevista, recurso flexible y dinámico. Investigación en educación médica, 2(7), 162-167. Recuperado en 24 de enero de 2019, de http://www.scielo.org.mx/ $\mathrm{s}$ c i e l o . p h p ? script=sci_arttext\&pid=S2007-5057201300030000 $9 \& \operatorname{lng}=$ es\&tlng=es.

23. Colombia. Ministerio de salud y protección social. (1993). Resolución 8430 de 1993. Por la cual se establecen las normas científicas, técnicas y administrativas para la investigación en salud. Bogotá: ministerio de salud y protección social.

24.Cantín, M. (2014). Declaración de Helsinki de la Asociación Médica Mundial: Principios éticos para las investigaciones médicas en seres humanos. Revisando su última versión. Int. J. Med. Surg. Sci, 1(4):339-346. https://doi.org/10.32457/ijmss. 2014.042

25.Gracia, E., \& Herrero, J. (2006). La comunidad como fuente de apoyo social. Evaluación e implicaciones en los ámbitos individual y comunitario.
Revista Latinoamericana de Psicología, 38, 327-342.

26.Boira, Santiago, Carbajosa, Pablo, \& Méndez, Raquel. (2016). Miedo, conformidad y silencio: la violencia en las relaciones de pareja en áreas rurales de Ecuador. Psychosocial Intervention, 25(1), 9-17. https://dx.doi.org/10.1016/j.psi.2015.07.008

27. Hewitt Ramírez, N., Juárez, F., Parada Baños, A. J., Guerrero Luzardo, J., Romero Chávez, Y. M., Salgado Castilla, A. M., \& Vargas Amaya, M. V. (2016). Afectaciones psicológicas, estrategias de afrontamiento y niveles de resiliencia de adultos expuestos al conflicto armado en Colombia. Revista Colombiana de Psicología, 25(1), 125-140.

28.Di-Colloredo, C., Aparicio, D., \& Moreno, J. (2007). Descripción de los estilos de afrontamiento en hombres y mujeres ante la situación de desplazamiento. Psychología. Avances de la Disciplina, 2(1), 125-156

29.Londoño, N., Patiño, C., Restrepo, D., Correa, J., Raigoza, J., Toro, L., ... Rojas, C. (2008). Perfil cognitivo asociado al trastorno por estrés postraumático en víctimas de violencia armada. Informes Psicológicos, 10, 11-27

30.Vasconcelles, E. (2005). Religious coping and psychological adjustment to stress: A meta-analysis. Journal of Clinical Psychology, 61(4), 461-480. doi: $10.1002 /$ jclp.20049. 